\title{
Descriptive study of functional or dysfunctional ruminative thoughts in patients with addictive disorders
}

\author{
Vincenti Marine $^{1 *}$,Faget-Agius Catherine ${ }^{1-4}$, Boyer Laurent ${ }^{2,4}$ and Lançon Christophe Ch-4 $^{1-4}$ \\ ${ }^{1}$ Department of Addictive disorders, Sainte Marguerite University Hospital, 13009 Marseille, France \\ ${ }^{2}$ Aix-Marseille University, EA 3279, 13005 Marseille, France \\ ${ }^{3}$ Public Health: Chronic Diseases and Quality of Life, School of Medicine, Timone University, 13005 Marseille, France \\ ${ }^{4}$ Department of Public Health, La Timone University Hospital, Assistance Publique - Hôpitaux de Marseille, 13005 Marseille, France
}

\begin{abstract}
Introduction: Ruminations are considered as a transdiagnostic factor that could explain the high co-morbidity between psychiatric and addictological pathologies. The main objective of our study is to evaluate the frequency of dysfunctional ruminative thoughts compared to functional thoughts in patients with addicted disorders, all addictions combined. The second objective is to compare the frequency of functional and dysfunctional ruminative thoughts within each addiction. The third objective is to study the correlations between the ruminative thoughts and anxiety and depression disorders in patients with addictive disorders.
\end{abstract}

Material and method: This is a cross-sectional descriptive epidemiological study. 89 patients from a referral center of addictive disorders were included in our study. We used two rumination questionnaires (Ruminative Response Scale and Mini Cambridge Exeter Ruminative Thought Scale)

Results: All addictions combined, there was a very significant difference between ruminations (functional or dysfunctional) for both scales. The same results are found for the alcohol, tobacco and behavioural addiction subgroups. For the opiate, hypnotic-sedative subgroups, there was a significant difference only for the Ruminative Response scale. No significant differences were found for the cannabis and cocaine groups.

Conclusion: In patients with addictive disorders, we found more dysfunctional thoughts than functional thoughts, in line with the transdiagnostic model of NolenHoeksema and Watkins. On the other hand, we reported differences between each addiction subgroups.

\begin{abstract}
Abbreviations: AAT: Abstract Analytical Thoughts; BDI: Beck Depression Inventory; CET: Concrete Experiential Thoughts; Mini CERTS: Cambridge Exeter Ruminative Thought Scale; RRS: Ruminative Response Scale; SCID: Structured Clinical Interview for DSM Disorder; STAI: Spielberger State Trait Anxiety Inventory
\end{abstract}

\section{Introduction}

In response style theory, rumination is defined as a mode of responding to distress, an coping strategy to control negative affect by repeatedly and passively focusing on negative symptoms, their causes and the possible consequences of them [1].

Rumination is a transdiagnostic factor. It would be a strategy of emotional regulation. If this strategy is inappropriate or dysfunctional, it can eventually lead to mental disorders $[2,3]$.

Studies suggest a significant influence of dysfunctional ruminative thoughts (Abstract Analytical Thoughts (AAT) and brooding) on alcohol consumption [4].

To this day, no study has looked at the frequency of ruminative thoughts in all addictions.

Our study is a continuation of the work of Nolen-Hoeksema, carried out in 2007, during a longitudinal study showing that brooding would be a risk factor for substance abuse [5].

The main objective of our study is to evaluate the frequency of dysfunctional ruminative thoughts compared to functional thoughts
(Concrete Experiential Thoughts (CET) and reflection) in patients with addicted disorders, all addictions combined.

The second objective is to compare the frequency of functional and dysfunctional ruminative thoughts within each addiction.

The third objective is to study the correlations between the ruminative thoughts and anxiety and depression disorders in patients with addictive disorders.

\section{Material and method}

\section{Study population}

This is a descriptive epidemiological study.

The inclusion of patients took place from January 1, 2018 to October 31,2018 at a referral center of addictive disorders (Sainte Marguerite University Hospital).

105 patients were included in the study.

${ }^{\star}$ Correspondence to: Marine Vincenti, Department of Addictive disorders, Sainte Marguerite University Hospital, France, E-mail: marine.vincenti@ap-hm.fr

Key words: addiction, anxiety, co-morbidities, depression, repetitive thinking, rumination

Received: May 28, 2019; Accepted: June 05, 2019; Published: June 10, 2019 
The inclusion criterion was to have at least two criteria belonging to a substance related or behaviour disorder at the time of assessment, based on the DSM 5 (Diagnostic and Statistical Manual of Mental Disorder) criteria determined by a psychiatrist after a diagnostic assessment.

The non-inclusion criteria were: (i) be under 18 years of age. (ii) be over 75 years of age.

The exclusion criteria were: (i) did not have any form of addiction (ii) had a current or past psychotic disorder. (iii) not have completed the entire diagnostic assessment and self-questionnaires.

15 patients were therefore excluded.

A total of 89 patients were studied in our study.

\section{Conduct of the study}

The study was unicentric. The participants were patients coming for a diagnostic and therapeutic evaluation at a referral center of addictive disorders (Sainte Marguerite University Hospital).

The study was approved by the Correspondent Informatique et Liberté de l'Assistance Publique des Hôpitaux de Marseille (APHM). All patients were informed orally that the data provided in the study would be processed after anonymization of the data and that participation in this research project was entirely voluntary.

\section{Questionnaires and scales used}

The SCID ("Structured Clinical Interview for DSM Disorder") diagnostic assessment questionnaire is a semi-structured interview conducted by a clinician. It includes 9 modules, i.e. 360 items (represented by open-ended questions) allowing 33 Axis I diagnoses to be identified according to the criteria of DSM 5 . It is based on the principle of a decision tree. [6]

The Spielberger State Trait Anxiety Inventory (STAI form Y) anxiety scale for adults is composed of two sub-ranges: Questionnaire YA: refers to the anxiety state felt when the participant answers it. Questionnaire YB: corresponds to the subject's usual tendency to be anxious [7]. (Appendix 1)

The Beck Depression Inventory (BDI 13) is the most widely used depression questionnaire in the adult population. It measures the level of depressive symptomatology of participants over the past 2 weeks. We used the 13-item version. Each item consists of 4 sentences of increasing intensity of a symptom. Each item varies between 0 and 3 points. The overall score is obtained by adding the scores of the 13 items (total score of 39) [8]. (Appendix 2)

We used two rumination evaluation questionnaires developed from the work of Watkins and Nolen-Hoeksema.

The first questionnaire is the Mini CERTS (Cambridge Exeter Ruminative Thought Scale). It includes 16 items. Likert scale, ranging from 1 (almost never) to 4 (almost always)). 7 items correspond to the CET against 9 that belong to the AAT. The total score varies between 16 and 64 [9]. (Appendix 3)

The second scale is the RRS ("Ruminative Response Scale"). The RRS distinguishes between these two types of ruminations: brooding and reflection Only 10 of the 22 items will be analyzed. Indeed, 12 items could be confused with the depressive symptoms themselves. Each item is evaluated on a Likert scale ranging from 1 (almost never) to 4 (almost always). The total score varies from 10 to 40 [10-12]. (Appendix 4)

\section{Statistical analyses}

The collected data were entered on Windows, Excel software. The statistical analysis was performed using the SPSS software [13].

The qualitative variables were described by proportions (absolute values and percentages). The continuous quantitative variables were measured using means (M) and standard deviation (SD). Comparisons between the subscales of the rumination questionnaires (brooding and reflection for the RRS and AAT and CET for the Mini CERTS) were made by the Student statistical test for averages (T-test). The materiality level was set at $\mathrm{p}<0.05$. We used a correlation test to analyze the rumination test data (RRS and Mini CERTS) with the depression (BDI) and anxiety (STAI) scales. The materiality level was set at $\mathrm{p}<0.05$.

\section{Results}

\section{Description of the population}

Among the 89 patients included in our study, the average age was 42.35 years (SD 13.97). 35 were women (39.5\%). 50 were in a couple (56.2\%), 43 of the participants had higher education (48.3\%) and 50 had children (56.2\%). Finally, 22 of them had had a judicial measure during their lifetime related to their addictions (24.7\%) (Table 1).

For the addiction subgroups, 54 of the participants had an alcohol use disorder (60.7\%). 66 a smoking disorder (74.2\%), 24 to cannabis (27.0\%), 10 to cocaine (11.2\%), 7 to opiates (7.9\%) and 7 to anxiolytic hypnotic sedatives (7.9\%). 18 participants had a behavioural addiction

Table 1. Socio-demographic and clinical characteristics of the study population

\begin{tabular}{|l|l|}
\hline Characteristics $\mathbf{n}=\mathbf{8 9}$ & \\
\hline socio demographic data & $42,35(13,970)$ \\
\hline age (years) M (SD) & \\
\hline gender & $35(39,5)$ \\
\hline women N (\%) & \\
\hline marital status & $50(56,2)$ \\
\hline couple N (\%) & \\
\hline education level & $43(48,3)$ \\
\hline university level N (\%) & \\
\hline parenthood & $50(56,2)$ \\
\hline children N (\%) & $22(24,7)$ \\
\hline judicial measures N (\%) & \\
\hline Clinical data & $54(60,7)$ \\
\hline alcohol use disorder N (\%) & $66(74,2)$ \\
\hline tobacco use disorder N (\%) & $24(27,0)$ \\
\hline cannabis use disorder N (\%) & $10(11,2)$ \\
\hline cocaine use disorder N (\%) & $7(7,9)$ \\
\hline opioid use disorder N (\%) & $7(7,9)$ \\
\hline sedative hypnotic anxiolytic use disorder N (\%) & $18(20,2)$ \\
\hline nonsubstance related disorder N (\%) & $38(42,7)$ \\
\hline antecedents of addiction N (\%) & $14(15,7)$ \\
\hline eating disorder N (\%) & $23,83(5,419)$ \\
\hline RRS M (SD) & $12,82(3,373)$ \\
\hline brooding M (SD) & $11,11(3,032)$ \\
\hline reflection M (SD) & $184,18(37,482)$ \\
\hline Mini CERTS M (SD) & $163,04(36,893)$ \\
\hline PAA M (SD) & $143,60(31,614)$ \\
\hline PCE M (SD) & $12,7(7,470)$ \\
\hline BDI score M (SD) & $96,48(20,763)$ \\
\hline STAI score M (SD) & $42,78(11,447)$ \\
\hline STAI YA M (SD) & $53,71(11,667)$ \\
\hline STAI YB M (SD) & \\
\hline & \\
\hline
\end{tabular}


(20.2\%). Finally, 38 were totally weaned from another addiction (42.7\%). 14 participants had a current or past eating disorder (15.7\%).

The average score of the RRS questionnaire was 23.83 (SD 5.419). Those of its two sub-ranges were 12.82 (SD 3.373) for brooding and 11.11 (SD 3.032) for reflection. For Mini CERTS, the total score averaged 306.64 (SD 52.783) and for subscales, the average for the AAT was 163.04 (SD 36.893) and the average for CET 143.60 (SD 31.614).

The average score of the BDI 13 was 12.7 (SD 7.470). The STAI average was 96.48 (SD 20.763). The average on the STAI YA subscale was 42.78 (SD 11.447) and STAI YB was 53.71 (SD 11.667).

Comparison of mean rumination questionnaire scores in the study population and for sub-samples of patients with addictive disorders

In the addictive population, all addictions combined, there was a very significant difference between the brooding and reflection scores $t$ $(0 ; 88)=4.64(p=0.000)$ as well as between the AAT and CET scores $\mathrm{t}$ $(0 ; 88)=4.17(p=0.000)($ Table 2$)$.

There was a significant difference between brooding and reflection for patients with an alcohol-related disorder t $(0 ; 54)=3.67(p=$ $0.001)$, a tobacco use disorder $\mathrm{t}(0 ; 65)=3.53(p=0.001)$, an opioid use disorder $\mathrm{t}(0 ; 6)=2.50(p=0.047)$, an anxiolytic sedative hypnotic use disorder $\mathrm{t}(0 ; 6)=2.82(p=0.030)$, a behavioural addiction $\mathrm{t}(0$; $17)=2.19(p=0.042)$ and for patients who have been withdrawn from another addiction.

There was no significant difference between brooding and reflection for patients with a cannabis use disorder $\mathrm{t}(0 ; 23)=0.90(p=0.373)$ and a cocaine-related use disorder $\mathrm{t}(0 ; 9)=-0.44(p=0.666)$.

There was a significant difference between AAT and CET for patients with an alcohol use disorders $\mathrm{t}(0 ; 53)=3.22(p=0.002)$, a tobacco use disorders $\mathrm{t}(0 ; 65)=3.29(p=0.002)$, a behavioural addiction $\mathrm{t}(0 ; 17)$ $=2.28(p=0.036)$.

There was no significant difference between AAT and CET for patients with a cannabis use disorder $\mathrm{t}(0 ; 23)=1,49(p=0.147)$, a cocaine-related use disorder $\mathrm{t}(0 ; 9)=-0.07(p=0.946)$, an opioid use disorder $\mathrm{t}(0 ; 6)=1,19(p=0.277)$, an anxiolytic hypnotic sedative use disorder $\mathrm{t}(0 ; 6)=1,50(p=0.183)$ and for patients withdrawn from other addiction $\mathrm{t}(0 ; 37)=1,52(p=0.135)$.

Table 2. Comparison of mean scores on rumination questionnaires in the study population and for sub-samples of patients with addictive disorders

\begin{tabular}{|l|l|l|}
\hline & RRS & Mini CERTS \\
\hline & Brooding vs Reflection & AAT vs CET \\
\hline Addictive disorder & t (p) & t (p) \\
\hline Alcohol use disorder & $4,64(0,000) * * *$ & $4,17(0,000)^{* * *}$ \\
\hline Tobacco use disorder & $3,67(0,001) * *$ & $3,22(0,002) * *$ \\
\hline Cannabis use disorder & $3,53(0,001)^{* *}$ & $3,29(0,002) * *$ \\
\hline Cocaine use disorder & $0,90(0,373)$ & $1,49(0,147)$ \\
\hline Opioid use disorder & $-0,44(0,666)$ & $-0,07(0,946)$ \\
\hline Sedative hypnotic anxiolytic use disorder & $2,50(0,47) *$ & $1,19(0,277)$ \\
\hline Nonsubstance related disorder & $2,82(0,030) *$ & $1,50(0,183)$ \\
\hline Antecedents of addiction & $2,19(0,042) *$ & $2,28(0,036) *$ \\
\hline & $4,21(0,000) * * *$ & $1,52(0,135)$ \\
\hline
\end{tabular}

RRS Ruminative Response Scale; Mini CERTS Mini Cambridge Exeter repetitive Thoughts; AAT Abstract Analytical Thoughts CET Concrete Experiential Thought $* \mathrm{p}<0,05 * * \mathrm{p}<0,01 * * * \mathrm{p}<0,001$
Correlation between rumination questionnaire scores and depression and anxiety questionnaires among the study population and sub-samples of patients with addictive disorders

The brooding subscale was very significantly and positively correlated with the reflection scores for patients with addicted disorders $\mathrm{r}=0.41(p=0.000)$; for patients with an alcohol-related disorder $\mathrm{r}=0.39$ $(p=0.003)$; a tobacco-related disorder $r=0.32(p=0.009)$ and for patients weaned from other addiction $r=0.65(p=0.000)$ (Table 3$)$.

There was no significant correlation between brooding and reflection for patients with cannabis use disorders, cocaine, opiates, anxiolytic hypnotic sedatives or those with behavioural addiction.

The brooding subscale was significantly and positively correlated with AAT scores for patients with addicted disorders $r=0.64(p=$ 0.000); for patients with an alcohol use disorder $r=0.59(p=0.000)$; a tobacco use disorder $\mathrm{r}=0.59 ;(p=0.000)$; a cannabis use disorder $\mathrm{r}=0.56(p=0.004) ;$ an opioid use disorder $\mathrm{r}=0.78(p=0.035) ;$ an anxiolytic sedative hypnotics use disorder $\mathrm{r}=0.93(p=0.002)$; a behavioural addiction $\mathrm{r}=0.65(p=0.003)$ and for patients weaned from another addiction $r=0.70(p=0.000)$.

There was no significant correlation between brooding and AAT for patients with cocaine use disorders.

The brooding subscale was significantly and positively correlated with BDI scores for patients with addicted disorders $r=0.37(p=0.000)$ ; for patients with an alcohol use disorder $r=0.39(p=0.003)$; a tobacco use disorder $r=0.33(p=0.005)$; a sedative hypnotic anxiolytic disorder $\mathrm{r}=0.86(p=0.012)$ and for patients withdrawn from other addiction $r$ $=0.43(p=0.006)$.

There was no significant correlation between brooding and BDI for patients with a disorder related to cannabis, cocaine, opiate use disorder and for patients with behavioural addiction.

The brooding subscale was significantly and positively correlated with STAI scores for patients with addicted disorders $r=0.38$ ( $p=$ $0.000)$; for patients with an alcohol use disorder $r=0.44(p=0.001)$; for patients with a tobacco use disorder $r=0.33(p=0.005)$; for patients with behavioural addiction $r=0.68(p=0.002)$ and for patients weaned from another addiction $r=0.49(p=0.002)$.

There was no significant correlation between brooding and STAI for patients with a disorder related to cannabis, cocaine, opiates and anxiolytic hypnotic sedatives.

The brooding subscale was significantly and positively correlated with STAI YA scores for patients with addicted disorders $\mathrm{r}=0.22(p=$ $0.032)$; for patients with alcohol-related disorders $r=0.310(p=0.023)$ ; an anxiolytic hypnotic sedative use disorders $r=0.85(p=0.013)$; for patients with a behavioural addiction $r=0.61(p=0.006)$.

There was no significant correlation between brooding and STAI YA for patients with a disorder related to tobacco use, cannabis, cocaine, opiates and anxiolytic hypnotic sedatives and for patients who were weaned from another addiction.

The brooding subscale was significantly and positively correlated with STAI YB scores for patients with addicted disorders $r=0.47(p=0.000)$; for patients with an alcohol use disorder $r=0.50(p=0.000)$; a tobacco use disorder $r=0.43(p=0.000)$; a behavioural addiction $r=0.52(p=0.024)$ and for patients weaned from other addiction $r=0.50(p=0.000)$. 
Table 3. Correlation between rumination questionnaire scores and depression and anxiety questionnaires among the study population and sub-samples of patients with addictive disorders

\begin{tabular}{|c|c|c|c|c|c|c|c|}
\hline & Reflection & AAT & CET & BDI & STAI & STAI YA & STAI YB \\
\hline & $\mathbf{r}$ & $\mathbf{r}$ & $\mathbf{r}$ & $\mathbf{r}$ & $\mathbf{r}$ & $\mathbf{r}$ & $\mathbf{r}$ \\
\hline \multicolumn{8}{|l|}{ Brooding } \\
\hline Addictions & $0.41 * *$ & $0.64 * *$ & 0.15 & $0.37 * *$ & $0.38 * *$ & $0.22 *$ & $0.47 * *$ \\
\hline Alcohol & $0.39 * *$ & $0.59 * *$ & -0.03 & $0.39 * *$ & $0.44 * *$ & $0.31 *$ & $0.50 * *$ \\
\hline Tobacco & $0.32 * *$ & $0.59 * *$ & 0.13 & $0.33 * *$ & $0.33 * *$ & 0.15 & $0.43^{* *}$ \\
\hline Cannabis & 0.23 & $0.56 * *$ & 0.36 & 0.18 & 0.08 & -0.08 & 0.24 \\
\hline Cocaine & 0.37 & 0.32 & 0.24 & -0.038 & -0.01 & 0.12 & -0.12 \\
\hline Opioid & 0.73 & 0.789* & 0.41 & 0.63 & 0.22 & -0.04 & 0.50 \\
\hline Sedative hypnotic anxiolytic & 0.60 & $0.93 * *$ & 0.19 & $0.86^{*}$ & 0.66 & $0.85^{* *}$ & 0.45 \\
\hline Behavioural addiction & 0.40 & $0.65 * *$ & -0.18 & 0.44 & $0.68 * *$ & $0.61 * *$ & $0.52 *$ \\
\hline Antecedents of addiction & $0.65^{* *}$ & $0.70 * *$ & 0.30 & $0.43 * *$ & $0.49 * *$ & 0.31 & $0.58 * *$ \\
\hline \multicolumn{8}{|l|}{ Reflection } \\
\hline Addictions & & $0.44 * *$ & $0.47 * *$ & 0.13 & 0.20 & 0.14 & $0.21 *$ \\
\hline Alcohol & & $0.40 * *$ & $0.32 *$ & 0.12 & $0.29^{*}$ & 0.18 & $0.35^{* *}$ \\
\hline Tobacco & & $0.28^{*}$ & $0.54 * *$ & -0.02 & 0.07 & 0.01 & 0.11 \\
\hline Cannabis & & 0.20 & $0.44 *$ & -0.16 & -0.66 & -0.06 & -0.05 \\
\hline Cocaine & & 0,20 & 0.17 & -0.26 & -0.04 & -0.03 & -0.04 \\
\hline Opioid & & $0.92 * *$ & 0.20 & 0.68 & 0.36 & 0.14 & 0.57 \\
\hline Sedative hypnotic anxiolytic & & 0.54 & 0.02 & 0.41 & 0.42 & 0.58 & 0.25 \\
\hline Behavioural addiction & & 0.34 & $0.48^{*}$ & 0.06 & 0.34 & $0.56^{*}$ & 0.09 \\
\hline Antecedents of addiction & & $0.60 * *$ & $0.58 * *$ & 0.33* & $0.33^{*}$ & $0.28)$ & $0.32 *$ \\
\hline \multicolumn{8}{|l|}{ PAA } \\
\hline Addictions & & & 0.18 & $0.53 * *$ & $0.55 * *$ & $0.38 * *$ & $0.60 * *$ \\
\hline Alcohol & & & 0.04 & $0.54 * *$ & $0.63 * *$ & $0.48 * *$ & $0.68^{* *}$ \\
\hline Tobacco & & & 0.17 & $0.49 * *$ & $0.55 * *$ & $0.38 * *$ & $0.60^{* *}$ \\
\hline Cannabis & & & 0.38 & $0.48^{*}$ & $0.52 * *$ & $0.43 *$ & $0.49 *$ \\
\hline Cocaine & & & -0.55 & 0.50 & 0.08 & -0.01 & 0.16 \\
\hline Opioid & & & 0.12 & 0.68 & 0.39 & 0.22 & 0.54 \\
\hline Sedative hypnotic anxiolytic & & & -0.13 & $0.96^{* *}$ & $0.84 * *$ & $0.97 * *$ & 0.65 \\
\hline Behavioural addiction & & & -0.07 & $0.83 * *$ & $0.81 * *$ & $0.54^{*}$ & $0.75^{* *}$ \\
\hline Antecedents of addiction & & & 0,13 & $0.63^{* *}$ & $0.62 * *$ & $0.45^{* *}$ & $0.67 * *$ \\
\hline \multicolumn{8}{|l|}{ PCE } \\
\hline Addictions & & & & -0.18 & -0.16 & -0.10 & -0.18 \\
\hline Alcohol & & & & -0.17 & -0.08 & -0.06 & -0.08 \\
\hline Tobacco & & & & -0.17 & -0.10 & -0.05 & -0.13 \\
\hline Cannabis & & & & 0.04 & 0.11 & 0.13 & 0.06 \\
\hline Cocaine & & & & -0.41 & 0.38 & 0.59 & 0.12 \\
\hline Opioid & & & & -0.36 & -0.34 & -0.51 & -0.11 \\
\hline Sedative hypnotic anxiolytic & & & & -0.18 & -0.56 & -0.26 & -0.69 \\
\hline Behavioural addiction & & & & $-3,32$ & -0.14 & 0.22 & -0.35 \\
\hline Antecedents of addiction & & & & -0.26 & -0.17 & -0.09 & -2.22 \\
\hline
\end{tabular}

PAA Pensées Analytiques Abstraites ; PCE Pensées Concrètes Expérientielles ; BDI Beck Depression Inventory ;

STAI State Trait Anxiety Inventory YA : état et YB : trait

$* * p<0.01 * p<0.05$

There was no significant correlation between brooding and STAI YB for patients with a disorder related to tobacco, cannabis, cocaine, opiates and anxiolytic hypnotic sedatives.

The reflection subscale was significantly and positively correlated with AAT scores for patients with addicted disorders $\mathrm{r}=0.44(p=$ $0.000)$; for patients with an alcohol use disorder $\mathrm{r}=0.50(p=0.000)$ ; a tobacco use disorder $r=0.28(p=0.019)$; an opioid use disorder $r$ $=0.92(p=0.003)$ and for patients weaned from another addiction $r=$ $0.60(p=0.000)$.

There was no significant correlation between reflection and AAT for patients with tobacco, cannabis, cocaine, and anxiolytic hypnotic sedatives disorders and for patients with behavioural addiction.

There is no significant correlation between reflection and BDI except for patients weaned from another addiction $\mathrm{r}=0.33(p=0.038)$.
There is no significant correlation between reflection and STAI except for patients with an alcohol use disorder $r=0.28(p=0.032)$ and patients weaned from another addiction $\mathrm{r}=0.33(p=0.038)$.

There is no significant correlation between reflection and STAI YA except for patients with a behavioural addiction $\mathrm{r}=0.56(p=0.014)$.

The reflection subscale was significantly and positively correlated with STAI YB scores for patients with addicted disorders $\mathrm{r}=0.21$ ( $p=$ $0.040)$; for patients with an alcohol use disorder $=0.35(p=0.009)$ and for patients weaned from other addiction $r=0.32(p=0.046)$.

There was no significant correlation between reflection and STAI YB for patients with a tobacco use disorder, cannabis, cocaine, opiates, anxiolytic hypnotic sedatives and for patients with behavioural addiction. 
The AAT subscale was significantly and positively correlated with BDI scores for patients with addicted disorders $r=0.53(p=0.000)$; for patients with an alcohol use disorder $r=0.54(p=0.000)$; a tobacco use disorder $\mathrm{r}=0.49(p=0.000)$; a cannabis use disorder $\mathrm{r}=0.48$ ( $p$ $=0.017) ;$ a sedative anxiolytic hypnotic disorder $r=0.961 ; \mathrm{p}=0.001 ;$ a behavioural addiction $r=0.83(p=0.000)$ and for patients who have been withdrawn from another addiction $r=0.63(p=0.000)$.

There was no significant correlation between AAT and BDI for patients with cocaine and opiate use disorders.

The AAT subscale was significantly and positively correlated with STAI scores for patients with a addicted disorders $r=0.55(p=0.000)$; for patients with an alcohol use disorder $r=0.63(p=0.000)$; a tobacco use disorder $\mathrm{r}=0.55(p=0.000)$; a cannabis use disorder $\mathrm{r}=0.52(p$ $=0.008)$; a sedative anxiolytic hypnotic disorder $\mathrm{r}=0.84(p=0.016)$; a behavioural addiction $\mathrm{r}=0.81(p=0.000)$ and for patients who have been withdrawn from another addiction $\mathrm{r}=0.62(p=0.000)$.

There was no significant correlation between AAT and STAI for patients with cocaine and opiate use disorders.

The AAT subscale was significantly and positively correlated with STAI YA scores for patients with addicted disorders $\mathrm{r}=0.38(p=0.000)$ ; for patients with an alcohol use disorder $r=0.486(p=0.000)$; a tobacco use disorder $r=0.381(p=0.002)$; a cannabis use disorder $r=$ $0.43(p=0.033)$; an anxiolytic hypnotic sedative use disorder $r=0.97(p$ $=0.000)$; a behavioural addiction $r=0.54(p=0.018)$ and for patients withdrawn from other addiction $r=0.45(p=0.004)$.

There was no significant correlation between AAT and STAI YA for patients with cocaine and opiate use disorders.

The AAT subscale was significantly and positively correlated with STAI YB scores for patients with addicted disorders $\mathrm{r}=0.60(p=$ $0.000)$; for patients with an alcohol use disorder $r=0.68(p=0.000)$ ; a tobacco use disorder $r=0.60(p=0.000)$; a cannabis use disorder $\mathrm{r}=0.49(p=0.013)$; a behavioural addiction $\mathrm{r}=0.75(p=0.000)$ and for patients who are weaned from another addiction $r=0.67(p=0.000)$.

There was no significant correlation between AAT and STAI YB for patients with cocaine, opiate and anxiolytic hypnotic sedative use disorders.

There is no significant correlation for the CET subscale for any other scale except the reflection subscale for patients with addicted disorders $r=0.47(p=0.000)$; for patients with an alcohol use disorder $\mathrm{r}=0.40(p=0.003)$; a tobacco use disorder $\mathrm{r}=0.54 p=(0.000) ; \mathrm{a}$ cannabis use disorder $\mathrm{r}=0.44(p=0.030)$; a behavioural addiction $\mathrm{r}=$ $0.48(p=0.043)$ and for patients withdrawn from other addiction $\mathrm{r}=$ $0.58(p=0.000)$.

\section{Discussion}

The purpose of this study was to evaluate and to compare the frequency between functional and dysfunctional ruminative thinking in a population of patients with addictive disorders.

As expected in our main hypothesis, patients with addictive disorders had more dysfunctional ruminative thoughts (AAT and brooding). Indeed, our sample of patients presents a large majority of patients with an alcohol use disorder, so it is consistent to find results similar to this subcategory. These results are consistent with the findings of Nolen-Hoeksema and Watkins [2].

We also showed that in patients with alcohol use disorders, dysfunctional ruminative thoughts (AAT and brooding) were more frequent than so-called functional thoughts (CET and reflection). These data are similar to the Caselli and Devynck studies. Indeed, many studies have focused on these patients and have shown that rumination is a predictor of alcohol consumption and of belonging to a pattern of consumption (problematic or social for example) $[4,14]$. These results are in line with the hypothesis that alcohol is a non-adaptive emotional regulation in the face of abstract ruminative thoughts [15]. They would intervene during all phases of consumption (at the time of craving, during alcohol consumption and after consumption on the resumption of alcohol consumption) [16]

Among patients with a smoking use disorder, we also find the same results as in the alcohol and general sample. AAT and brooding are more frequent than CET and reflection, respectively. The combination of these two addictions is very common. It is therefore not surprising to have similar results. To our knowledge, no study has studied in a more specific way the role of ruminations in tobacco use addiction. On the other hand, two studies find a positive link between nicotine addiction and certain metacognitions (positive beliefs about worry, negative beliefs about worry about their uncontrollability and dangers, and beliefs about cognitive trust) [17,18].

Among patients with a cannabis-related use disorder, no significant differences were found between AAT and CET, nor between brooding and reflection. Yet chronic cannabis use creates an amotivational syndrome that leads to a "no problem solving" attitude. This resembles in many ways the consequences of abstract analytical ruminative thoughts [19]. In addition, almost all cannabis smokers have a concomitant dependence on tobacco, due to the way it is used (mainly smoking). We could have expected to see similar results for the tobacco group, which is not the case.

Among patients with a cocaine-related use disorder, no significant differences were observed. This is the first study that addresses ruminations in patients with cocaine use disorders. Only one study links metacognitive deficits (the correspondence between objective performance and reported confidence in that performance) with cocaine use. They show that patients have significant impairment of perceptual neurocognitive functions. A second study focuses on emotional intelligence, where cocaine addicts are reported to have an increased and persistent inability to regulate negative mood following stress [20]. We can draw a parallel with the mechanisms of action of ruminative thoughts [21].

Among patients with opioid-related substance use disorders, brooding were more frequent than reflection. No significant difference was found for the Mini CERTS questionnaire. Only one study treated negative metacognitions in patients with chronic pain using opioids. The use of opioids could be a non-adaptive therapeutic strategy for the management of chronic pain and metacognitions in these patients [22].

Among patients with a use disorder related to sedatives, hypnotics and anxiolytics, brooding were more frequent than reflection. No significant difference was found for the Mini CERTS subscales. Anxiety addiction frequently begins with a prescription for benzodiazepines following a sleep disorder. Preliminary studies have shown that the main complaint of patients with sleep disorders was their inability to stop or control their thoughts. Indeed, in his patients the rates of dysfunctional ruminations were higher [23]. Disruptions in polysomnography were found in these patients (the presence of dysfunctional repetitive thoughts was associated with shorter total subjective sleep time, lower subjective sleep quality and increased perception of disturbed sleep). The place of therapies targeting concerns or ruminative thoughts 
could then be an alternative to the prescription of a hypnotic such as benzodiazepines and thus avoid the risk of our patients developing a psychological and physical dependence on this substance [24,25].

Among patients with behavioural addiction, dysfunctional ruminative thoughts (AAP and brooding) were much more frequent than so-called functional thoughts (AAP and reflexion). No studies deal with ruminative thoughts per se, but 4 deal with metacognitions and pathological gambling. These studies have shown that patients with gambling addiction have higher levels of metacognitions related to the "need to control their thoughts". It would even be a predictor of the level of severity of pathological gambling. Moreover, anxiety and depression symptomatology would therefore contribute to the establishment of beliefs that would encourage gambling. [26-30].

On the other hand, many studies have focused on the link between ruminative thinking and Eating Disorders The concept of food addiction is nowadays increasingly mentioned in the context of eating disorders (Eating disorders with eating compulsions (anorexia-bulimia, bulimia, bulimia or binge eating).

Among patients with withdrawal from another addiction (42.7\%), there is a very significant difference between brooding and reflection, which is not the case between AAT and CET. It has been shown in studies of the alcohol-dependent population that the presence of dysfunctional ruminations is a predictor of relapse [31].

The analysis of correlations in the population with an alcohol use disorder is similar to previous studies. The brooding showed a very significant positive correlation with the AAT. AAT and brooding were correlated with BDI, STAI (total, and its status and trait forms). These results are related to Watkins' results which postulate that abstractanalytical thinking is a dysfunctional mode of repetitive thinking. The CET did not correlate with the BDI or STAI, nor with the AAT or brooding [32]. The CET, on the other hand, were correlated with the reflection. However, our study did not show a negative correlation as in Devynck's study with BDI or STAI. These results are consistent with Watkins' hypothesis that concrete-experiential thinking could be a more appropriate repetitive way of thinking. Our study shows a positive correlation of reflection with brooding and AAT and STAI (total and its form YB trait) [32]. She had no connection to the BDI. These results are similar to Devynck's study and challenge Treynor's idea of reflection as an adaptive form of ruminative thinking with positive consequences. $[4,11]$.

The same results are found in the population with a substance use disorder and in the sub-population of tobacco use disorders. These results are not surprising given that most patients with alcohol use disorders very frequently have a concurrent smoking disorder. Our sample is similar to the general population. Studies of patients with alcohol use disorders do not specify whether they have a tobacco addiction or an alcohol-only disorder. Regarding the sample with a cannabis use disorder, the results are less important. AAT are positively and significantly correlated with BDI, STAI and brooding. This is not the case with the BDI and STAI. The reflection and CET were not linked to brooding, AAT, BDI and STAI. On the other hand, they were positively and significantly correlated with each other. Cannabis smokers also smoke tobacco (associated consumption pattern), so the results are out of step with those of the tobacco sample and would require further studies on a larger sample. No correlation was shown in the sample with a cocaine-related disorder that consisted of only 10 patients. For the group with an opioid use disorder", the brooding showed a link with the BDI, the STAI. The AAT did not show a link with the BDI and STAI. The reflection was also positively correlated with the AAT. The other values had no significant relationship between them. Among patients with an anxiolytic use disorder, one particularity is observed: brooding and AAT are positively and significantly correlated with STAI YA (state) and not with STAI (total or YB trait). Brooding and AAT are also correlated with each other. One of the avenues of research for future studies could be the research in its patients of an emotional regulation by anxiolytic treatments of an immediate appeasement (STAI YA condition) and not of their background anxiety (STAI YB trait) which could be a reason that they are more at risk of developing an addiction to these substances. Correlations among the behavioural addiction sub-sample have a particularity. The downgrading did not show a significant link with the BDI, which was the case for the AAT and BDI. Another specificity was that the reflection was positively and significantly correlated with STAI YA and PCE. For the rest, brooding and AAT were positively and significantly correlated with STAI. No other values were related to CET and reflection. Finally, for the group weaned from another addiction, the AAT and brooding were positively and significantly correlated with each other, as well as with the BDI and STAI. The reflection was positively and significantly correlated with brooding, AAT and CET. CET had no other significant correlation.

Some limitations of this study must be recognized.

One of the limitations of our study is the absence of a control group.

We included all patients with a substance use disorder after assessment by a psychiatrist underpinned by the interview with SCID (heterostructured interview questionnaire).

Second, the sample is made up of more men than women. The literature showed that rumination was more related to depression in women than in men and that rumination explained the gender difference in depression. Nolen-Hoeksema and Harrell showed that rumination was a significant predictor of alcohol problems in women, but not in men [33]. The proportion of men compared to women in our sample may explain why some results are not significant [34].

Third, as in previous studies, rumination was assessed with selfreported measures. Although we used validated questionnaires, the questionnaires are limited by biases (retrospective recall bias, social desirability bias and lack of precision) [35].

Finally, the small size of our sub-samples, their lack of independence due to the fact that they are linked by addictive comorbidities, do not allow us to draw conclusions about the influence of ruminative thoughts in these populations.

Our study is the first on a population with a substance use or behavioural disorder

Further studies should be developed to support its initial results.

\section{Conclusion}

Our study is a preliminary study, which opens up the perspective of new research in addictology. The results are in line with the studies conducted in alcohol-dependent people. Patients with a substance use or behaviour disorder would have more dysfunctional ruminative thoughts (AAT and brooding) than those described as adaptive (CET and reflection). This difference is found for patients with alcohol, tobacco and behavioural addiction disorders. There is only a significant difference between the brooding and reflection scores for patients with opioid-related use disorders, anxiolytic sedative hypnotics and patients who have been weaned from another addiction. 


\section{Acknowledgements}

We would like to thank the referral center of addictive disorders (Sainte Marguerite University Hospital) in the Bouches-du-Rhône, France, as well as the heads of the medical department, Professors Christophe Lançon, Laurent Boyer and Dr Catherine Faget-Agius. We also thank nurses Nathalie Bochu, Marie-Paule Bonetti and Martine Garrido for their help in collecting data.

\section{Conflict of interest}

All authors declare that they have no conflict of interest.

\section{Acknowledgement}

This research has not received any specific funding from public, commercial or not-for-profit funding agencies.

\section{References}

1. Nolen-Hoeksema S, Wisco BE, Lyubomirsky S (2008) Rethinking Rumination. Perspect Psychol Sci 3: 400-424. [Crossref]

2. Nolen-Hoeksema S, Watkins ER (2011) A Heuristic for Developing Transdiagnostic Models of Psychopathology: Explaining Multifinality and Divergent Trajectories. Perspect Psychol Sci 6: 589-609. [Crossref]

3. Aldao A, Nolen-Hoeksema S, Schweizer S (2010) Emotion-regulation strategies across psychopathology: A meta-analytic review. Clin Psychol Rev 30: 217-237. [Crossref]

4. Devynck F, Kornacka M, Sgard F, Douilliez C (2017) Repetitive Thinking in AlcoholDependent Patients. Subst Use Misuse 52: 108-118. [Crossref]

5. Nolen-Hoeksema S, Stice E, Wade E, Bohon C (2007) Reciprocal relations between rumination and bulimic, substance abuse, and depressive symptoms in female adolescents. J Abnorm Psychol 116: 198-207. [Crossref]

6. First MB, Spitzer RL, Gibbon M, Williams JB (2002) Structured Clinical Interview for DSM-IV-TR Axis I Disorders, Research Version, Patient Edition (SCID-I/P). Biometrics Research - State Psychiatric Institute.

7. Spielberger CD (1989) State-Trait Anxiety Inventory: a comprehensive bibliography. Palo Alto, CA: Consulting Psychologists Press.

8. Alsaleh M, Lebreuilly R (2017) Validation de la traduction française d'un questionnaire court de dépression de Beck (BDI-FS-Fr). Annales Médico-psychologiques, revue psychiatrique 175: 608-616.

9. Douilliez C, Heeren A, Lefèvre N, Watkins E, Barnard P, et al. (2014) Validation de la version française d'un questionnaire évaluant les pensées répétitives constructives et non constructives. Canadian Journal of Behavioural Science / Revue canadienne des sciences du comportement 46: 185-192.

10. Nolen-Hoeksema S, Morrow J (1991) A Prospective Study of Depression and Posttraumatic Stress Symptoms After a Natural Disaster: The 1989 Loma Prieta Earthquake. J Pers Soc Psychol 61: 115-121. [Crossref]

11. Treynor W, Gonzalez R, Nolen-Hoeksema S (2003) Rumination Reconsidered: A Psychometric Analysis. Cognitive Therapy and Research 13.

12. Parola N, Zendjidjian XY, Alessandrini M, Baumstarck K, Loundou A, et al. (2017) Psychometric properties of the Ruminative Response Scale-short form in a clinical sample of patients with major depressive disorder. Patient Prefer Adherence 11: 929937. [Crossref]

13. IBM Corp. Released (2015) IBM SPSS Statistics for Windows. IBM Corp. (s. d.). Armonk, NY.

14. Caselli G, Bortolai C, Leoni M, Rovetto F, Spada MM (2008) Rumination in problem drinkers. Addiction Research \& Theory 16: 564-571.

15. Baeyens C, Kornacka M, Douilliez C (2012) De la dépression à une perspective transdiagnostique conceptualisation et interventions sur les ruminations mentales (s.d.).

16. Spada MM, Caselli G, Wells A (2013) A Triphasic Metacognitive Formulation of Problem Drinking: A Triphasic Metacognitive Formulation of Problem Drinking. Clin Psychol Psychother 20: 494-500. [Crossref]

17. Nikcevic AV, Spada MM (2008) Metacognitions Across the Continuum of Smoking Dependence. Behavioural and Cognitive Psychotherapy 36: 333-339.
18. Spada MM, Nikcevic AV, Moneta GB, Wells A (2007) Metacognition as a mediator of the relationship between emotion and smoking dependence. Addict Behav 32: 2120 2129. [Crossref]

19. Hyman SM, Sinha R (2009) Stress-related factors in cannabis use and misuse: Implications for prevention and treatment. J Subst Abuse Treat 36: 400-413. [Crossref]

20. Moeller SJ, Fleming SM, Gan G, Zilverstand A, Malaker P, et al. (2016) Metacognitive impairment in active cocaine use disorder is associated with individual differences in brain structure. Eur Neuropsychopharmacol 26: 653-662. [Crossref]

21. Fox HC, Bergquist KL, Casey J, Hong KA, Sinha R (2010) Selective Cocaine-Related Difficulties in Emotional Intelligence: Relationship to Stress and Impulse Control Emotional Intelligence in Cocaine Dependent Individuals. The American Journal on Addictions.

22. Ziadni MS, Sturgeon JA, Darnall BD (2018) The relationship between negative metacognitive thoughts, pain catastrophizing and adjustment to chronic pain. Eur $J$ Pain 22: 756-762. [Crossref]

23. Galbiati A, Giora E, Sarasso S, Zucconi M, Ferini-Strambi L (2018) Repetitive though is associated with both subjectively and objectively recorded polysomnographic indices of disrupted sleep in insomnia disorder. Sleep Med 45: 55-61. [Crossref]

24. Zoccola PM, Dickerson SS, Zaldivar FP (2008) Rumination and Cortisol Responses to Laboratory Stressors. Psychosom Med 70-661-667. [Crossref]

25. Shrivastava D, Jung S, Saadat M, Sirohi R, Crewson K (2014) How to interpret the results of a sleep study. J Community Hosp Intern Med Perspect p. 4.

26. Hamonniere T, Varescon I (2018) Implication des croyances métacognitives dans les conduites addictives?: point sur les connaissances. Journal de Thérapie Comportementale et Cognitive 28: 80-92.

27. Spada MM, Roarty A (2015) The relative contribution of metacognitions and attentional control to the severity of gambling in problem gamblers. Addict Behav Rep 1: 7-11. [Crossref]

28. Lindberg A, Fernie BA, Spada MM (2011) Metacognitions in problem gambling. $J$ Gambl Stud 27: 73-81. [Crossref]

29. Mansueto G, Pennelli M, De Palo V, Monacis L, Sinatra M, et al. (2016) The Role of Metacognition in Pathological Gambling: A Mediation Model. J Gambl Stud 32: 93-106. [Crossref]

30. Jauregui P, Urbiola I, Estevez A (2016) Metacognition in Pathological Gambling and Its Relationship with Anxious and Depressive Symptomatology. J Gambl Stud 32: 675688. [Crossref]

31. Caselli G, Ferretti C, Leoni M, Rebecchi D, Rovetto F, et al. (2010) Rumination as a predictor of drinking behaviour in alcohol abusers: a prospective study: Rumination as a predictor of drinking behaviour. Addiction 105: 1041-1048. [Crossref]

32. Watkins E (2004) Adaptive and maladaptive ruminative self-focus during emotional processing. Behav Res Ther 42: 1037-1052. [Crossref]

33. Nolen-Hoeksema S, Harrell ZA (2002) Rumination, Depression, and Alcohol Use: Tests of Gender Differences. 16.

34. Nolen-Hoeksema S (1987) Sex differences in unipolar depression: evidence and theory. Psychol Bull 101: 259-282. [Crossref]

35. Serre F, Fatseas M, Swendsen J, Auriacombe M (2015) Ecological momentary assessment in the investigation of craving and substance use in daily life: A systematic review. Drug Alcohol Depend 148: 1-20. [Crossref]

Copyright: (C2019 Marine V. This is an open-access article distributed under the terms of the Creative Commons Attribution License, which permits unrestricted use, distribution, and reproduction in any medium, provided the original author and source are credited. 